\title{
CORRELAÇÃO ENTRE COMPOSTOS BIOATIVOS E ATIVIDADE ANTIOXIDANTE DE POLPA DE GUAPEVA (Pouteria cf. Guardneriana Radlk)
}

Correlation between bioactive compounds and antioxidant activity of guapeva pulp (Pouteria cf. Guardneriana Radlk)

Correlación entre compuestos bioactivos y actividad antioxidante de la pulpa de guapeva (Pouteria cf. Guardneriana Radlk)

Camila Mariane da Silva Soares ${ }^{* 1,3}$, Rômulo Alves Morais ${ }^{1,3}$, Romilda Ramos da Silva ${ }^{1}$, Aynaran Oliveira de Aguiar $^{3}$, André Leonardo dos Santos ${ }^{1,3}$, Andréia Ibiapina ${ }^{3}$, Glêndara Aparecida de Souza Martins ${ }^{1}$

${ }^{1}$ Laboratório de Cinética e Modelagem de Processos (LaCiMP) - Universidade Federal do Tocantins.

${ }^{2}$ Programa de Pós-Graduação em Ciência e Tecnologia de Alimentos (PPGCTA -UFT) - Universidade Federal do Tocantins.

${ }^{3}$ Mestre em Ciência e Tecnologia de Alimentos - Universidade Federal do Tocantins.

*Correspondência: Laboratório de Cinética e Modelagem de Processos, Escola de Engenharia de Alimentos, Universidade Federal do Tocantins, Quadra 109 Norte, Avenida NS 15, ALCNO-14, Plano Diretor Norte, Palmas -TO, CEP: 77001-090.e-mail: camisoares06@gmail.com.

\section{Artigo recebido em 18/02/2020 aprovado em 23/03/2020 publicado em 26/03/2020.}

\section{RESUMO}

A guapeva (Pouteria cf. Guardneriana Radlk) é um fruto oriundo do cerrado, pouco conhecido, mas que possui alto potencial fenolico, como carotenoides, vitamina $\mathrm{C}$ e comprovada atividade antioxidante, que pode contribuir no combate a doenças crônico-degenerativas, tais como câncer e diabetes. Os compostos fenolicos agem de forma antioxidante combatendo os radicais livres reagindo com DNA, RNA, proteínas e outras substâncias oxidáveis. Assim o objetivo desta pesquisa foi verificar as correlações entre os compostos bioativos da polpa de guapeva e os compostos antioxidantes utilizando a Correlação de Pears. Verificou-se que o conteúdo fenólico presente na polpa da guapeva apresentou forte correlação com vitamina $C(R=0,99)$ e carotenóide $(R=0,88)$, demosntrando que esses constituintes contribuem de forma expressiva no potencial fenólico do fruto, e os carotenóides totais correlacionouse fortemente com as antocianinas e o flavonoide $(\mathrm{R}=0,97$ e $\mathrm{R}=0,91)$. Para os resultados entre conteúdo fenólico e o método de FRAP demonstrou correlação considerada moderada $(\mathrm{R}=0,59)$ atestando um vínculo estreito entre os compostos fenólicos e FRAP, indicando que a capacidade antioxidante da guapeva tem associação com os seus constituintes fenólicos. Ainda assim o fruto é considerado como um alimento que possui um alto potencial antioxidante natural podendo ser incorporado na dieta humana.

Palavras-chave: potencial antioxidante, frutos, cerrado.

\section{ABSTRACT}

Guapeva (Pouteria cf. Guardneriana Radlk) is a fruit from the Cerrado, little known, but has high phenolic potential, such as carotenoids, vitamin $C$ and proven antioxidant activity, which can contribute to the fight against chronic degenerative diseases such as cancer and diabetes. Phenolic compounds act antioxidant by fighting free radicals by reacting with DNA, RNA, proteins and other oxidizable substances. Thus the objective of this research was to verify the correlations between bioactive compounds of guapeva pulp and antioxidant compounds using the Pears Correlation. The phenolic content present in guapeva pulp was strongly correlated with vitamin $C(R=0.99)$ and carotenoid $(R=0.88)$, demonstrating that these constituents contribute significantly to the phenolic potential of the 
fruit, and total carotenoids correlated strongly with anthocyanins and flavonoid $(R=0.97$ and $R=0.91)$. For the results between phenolic content and the FRAP method showed a moderate correlation $(R=0.59)$, attesting to a close bond between phenolic compounds and FRAP, indicating that guapeva antioxidant capacity is associated with its phenolic constituents. Still the fruit is considered as a food that has a high natural antioxidant potential and can be incorporated into the human diet.

Keywords: antioxidant potential, fruits, Cerrado.

\section{RESUMEN}

La guapeva (Pouteria cf. Guardneriana Radlk) es una fruta del Cerrado, poco conocida, pero tiene un alto potencial fenólico, como los carotenoides, la vitamina $C$ y la actividad antioxidante comprobada, que puede contribuir a la lucha contra las enfermedades degenerativas crónicas como el cáncer. y diabetes Los compuestos fenólicos actúan como antioxidantes al combatir los radicales libres al reaccionar con DNA, RNA, proteínas y otras sustancias oxidables. Por lo tanto, el objetivo de esta investigación fue verificar las correlaciones entre los compuestos bioactivos de la pulpa de guapeva y los compuestos antioxidantes utilizando la correlación de peras. El contenido fenólico presente en la pulpa de guapeva se correlacionó fuertemente con la vitamina $C(R=0,99)$ y el carotenoide $(R=0,88)$, lo que demuestra que estos componentes contribuyen significativamente al potencial fenólico de la fruta, y los carotenoides totales se correlacionaron fuertemente con antocianinas y flavonoides $(R=0.97$ y $R=0.91)$. Los resultados entre el contenido fenólico y el método FRAP mostraron una correlación moderada $(R=0.59)$ que atestigua un vínculo estrecho entre los compuestos fenólicos y FRAP, lo que indica que la capacidad antioxidante de la guapeva está asociada con sus componentes fenólicos. Aún así, la fruta se considera como un alimento que tiene un alto potencial antioxidante natural y puede incorporarse a la dieta humana.

Descriptores: Potencial antioxidante, frutas, cerrado.

\section{INTRODUÇÃO}

Os frutos nativos do cerrado são conhecidos por possuírem sabores únicos, logo são denominados como frutas exóticas. Estudos já apontam que além de serem consumidos in natura, o potencial econômico desses frutos vem crescendo, tanto na produção de alimentos como no reconhecimento de seu potencial farmacológico e propriedades nutracêuticas (MOTA et al., 2015).

Entre as diversas espécies de frutos nativos desse bioma, os pioneiros em interesse econômico e social já estão sendo largamente estudados, entre eles estão o baru, mangaba e buriti, por exemplo. Porém o cerrado possui um vasto número de espécies de frutos que possuem estudos escassos como referência, entre eles destaca-se a Guapeva (Pouteria cf guardneriana Radlk) (MOREIRA-ARAÚJO et al., 2019).

A caracterização física e química de frutos nativos do cerrado e a quantificação de componentes bioativos são importantes para o reconhecimento do valor nutricional, e do ponto de vista comercial, para agregar valor e qualidade ao produto final. O consumo desses frutos tem sido associado à diminuição da incidência de mortalidade por doenças crônicas devido aos altos níveis de compostos bioativos que proveem benefícios a saúde (CANUTO et al., 2010).

Graças a uma alta atividade antioxidante características dos frutos oriundos do cerrado, uma diversidade de compostos fenólicos exerce uma função importante nos processos de redução de riscos de doenças cardiovasculares podendo ainda atuar no estresse oxidativo relacionado a doenças como, diabetes, arteriosclerose e doenças neurodegenerativas (ROCHA et al., 2011; ASADI et al., 2010).

A atividade antioxidante de comostos fenólicos deve-se, principalmente, às propriedades redutoras e estrutura química que possuem. Essas características realizam uma função importante na neutralização de radicais livres e quelação de metais de transição, atuando na etapa de iniciação e no desenvolvimento do 
processo oxidativo (ABRAHÃO et al., 2010). Esses compostos são associados com frequência a inibição do crescimento de células cancerígenas, a ação antiinflamatória e antimicrobiana (JARA-PALACIOS et al., 2015; LEE et al., 2006; VU et al., 2012; LAJILI et al., 2016; CETIN-KARACA; NEWMAN, 2015).

Antioxidantes são então substâncias que quando estão presentes em pequenas concentrações, diminuem de forma significante ou cessam a oxidação do substrato. Possuem a função de reduzir os danos causados pela ação dos radicais livres e/ou espécies reativas não radicais (BARBOSA et al., 2010).

$\mathrm{O}$ presente estudo tem como objetivo analisar as correlações entre os compostos bioativos da polpa de guapeva e os compostos antioxidantes utilizando a Correlação de Pearson.

\section{MATERIAIS E MÉTODOS}

Foram utilizadas polpas de frutos de guapeva (Pouteria cf. Guardneriana Radlk) maduros, colhidos no cerrado local da cidade Palmas-TO. A análise de correlação linear (correlação de Pearson) foi realizada entre as médias dos resultados referentes aos compostos bioativos (compostos fenólicos totais, vitamina $\mathrm{C}$, carotenoides totais, antocianinas, flavonoides amarelos,) e a atividade antioxidante total (dos métodos $\mathrm{DPPH}^{*}$ e FRAP) da polpa do fruto da guapeva a nível de significância de $p \leq 0,05$.

Para a determinação de composto fenólicos totais, utilizou-se a matéria fresca conforme o método espectrofotométrico Folin-Ciocalteau descrito por Singleton (1965), os resultados foram expressos em $\mathrm{mg}$ GAE g-1.

$\mathrm{O}$ teor de Vitamina $\mathrm{C}$ foi determinado de acordo com a metodologia do Instituto Adolfo Lutz (2008), por titulação das amostras foram em solução de iodato de potássio a $0,02 \mathrm{M}$ até a obtenção da coloração azul. $\mathrm{O}$ resultado foi expresso em $\mathrm{mg}$ de vitamina $\mathrm{C}$ por $100 \mathrm{~g}$ de fruta.

A quantificação e determinação dos carotenoides totais seguiu a metodologia espectrofotométrica posposta por Higby (1962). A absorbância foi medida a $450 \mathrm{~nm}$ e os resultados expressos em mg de carotenoides totais por $100 \mathrm{~g}$ de amostra.

As análises de antocianinas e flavonoides amarelos foram realizadas conforme descrito por Francis (1982). Os resultados foram expressos em $\mu \mathrm{g}$ por $100 \mathrm{~g}^{-1}$, calculados através das fórmulas: ANT = fator de diluição $\mathrm{x}$ absorbância/98,2 FLV = fator de diluição x absorbância/76,6.

A atividade de captura do radical livre foi medida pelo método DPPH, o qual baseia-se na redução deste radical livre, relativamente estável, DPPH (2,2 difenil-1-picrilhidrazila), em solução etanol/água 8:2 (EtOH). Seguindo a metodologia descrita por Brand-Willians et al. (1995) modificada por Rufino et al. (2007), onde a coloração violeta do radical DPPH é reduzida por substâncias antioxidantes e sua cor muda para amarelo, passando para sua forma estável, DPPH-H. Os resultados de $\mathrm{EC}_{50}$ foram expressos em g de fruto/g de DPPH.

A atividade antioxidante avaliada quanto ao poder de redução do Ferro (FRAP) foi medida pelo método de Rufino et al. (2006), em solução em etanol/água 8:2 (EtOH). O potencial antioxidante dos extratos da polpa de guapeva foi determinado baseado na curva de calibração, traçada usando Sulfato Ferroso $2 \mathrm{mM}$ em concentrações que variaram entre $500 \mathrm{e}$ $2.000 \mathrm{mM}$. Os resultados foram expressos em $\mu \mathrm{M}$ sulfato ferroso/g de fruta.

Foi realizada uma análise multivariada (ACP) dos componentes principais, utilizando a média dos resultados para cada parâmetro avaliado na polpa de 
guapeva, afim de verificar o agrupamento das diferentes respostas dos seus constituintes bioativos e atividade antioxidante, e assim simular como é a relação entre essas variáveis (VÍTOLO et al., 2012).

Com o intuito de avaliar a relação entre a variáveis estudadas, adotou-se a correlação de Pearson, na qual indica a existência positiva ou negativa entre duas variáveis, para verificar a significância foi adotado $\alpha=5 \%$ (coeficiente de correlação). Para o tratamento dos dados utilizou-se o software Statistica 7.0, adotando como critério de absorção mínima um coeficiente de correlação acima de $70 \%$, o que indicando uma forte relação entre as variáveis avaliadas (BURGARD e KUZNICKI, 2018).

\section{RESULTADOS E DISCUSSÃO}

Através da correlação de Person descritas na Tabela 1 as verificações das interações serão avaliadas conforme os critérios descrito por Figueiredo Filho e Silva Junior (2009), como: $(\mathrm{r}=1,00)$ perfeita, $(0,80 \leq \mathrm{r}$ $<1,00)$ forte, $(0,50 \leq \mathrm{r}<0,80)$ moderada e $(0,10 \leq \mathrm{r}<$ $0,50)$ fraca.

$\mathrm{Na}$ Tabela 1 observa-se que existe uma correlação entre os compostos fenólicos e teor de vitamina $C(R=0,99)$ apresentados graficamente na Figura 1 e carotenoides totais $(\mathrm{R}=0,88)$ na Figura 2, ambas consideradas fortes, demonstrando que estes são constituintes importante para o potencial fenólico da guapeva. Vários estudos mostraram alta correlação entre o conteúdo de compostos fenólicos e teor Vitamina C (CONTRERÁS-CALDERÓN et al. 2011; RUFINO et al. 2010).

Figura 1: Gráficos de Correlação entre o conteúdo de Compostos Fenólicos e Vitamina C



A contribuição da vitamina $\mathrm{C}$ para a atividade antioxidante das frutas é altamente variável (SOUZA et al., 2018). Além disso fatores edafoclimáticos podem interferir no conteúdo de compostos bioativos dos frutos, tais como: Compostos fenólicos, vitamina $\mathrm{C}$ e carotenoide (MOREIRA-ARAUJO et al., 2019).

Tabela 1: Coeficientes de correlação entre os teores de compostos fenólicos, vitamina C, carotenóides totais, flavonoides amarelos, e atividade antioxidante (DPPH e FRAP) para as polpa de Guapeva (Pouteria cf. Guardneriana Radlk).

\begin{tabular}{|c|c|c|c|c|c|c|c|}
\hline VARIÁVEIS & $\begin{array}{c}\text { Comp. } \\
\text { Fenólicos }\end{array}$ & $\begin{array}{c}\text { Vitamina } \\
\mathrm{C}\end{array}$ & $\begin{array}{c}\text { Carotenoides } \\
\text { Totais } \\
\end{array}$ & Antocianinas & $\begin{array}{c}\text { Flavonoides } \\
\text { Amarelos }\end{array}$ & DPPH & FRAP \\
\hline Comp. Fenólicos & 1 & & & & & & \\
\hline Vitamina C & $0,996^{*}$ & 1 & & & & & \\
\hline Carotenóides Totais & $0,880 *$ & $0,836^{*}$ & 1 & & & & \\
\hline Antocianinas & $0,757^{*}$ & 0,698 & $0,976^{*}$ & 1 & & & \\
\hline $\begin{array}{c}\text { Flavonoides } \\
\text { Amarelos }\end{array}$ & 0,620 & 0,551 & $0,918^{*}$ & $0,982^{*}$ & 1 & & \\
\hline DPPH & 0,187 & 0,270 & 0,301 & $-0,500$ & $-0,654$ & 1 & \\
\hline FRAP & 0,597 & 0,376 & 0,179 & 0,311 & 0,201 & 0,322 & 1 \\
\hline
\end{tabular}


*Valores de correlação significativos para $\alpha=5 \%$.

Figura 2: Gráficos de Correlação entre o conteúdo de Compostos Fenólicos e Carotenoides.

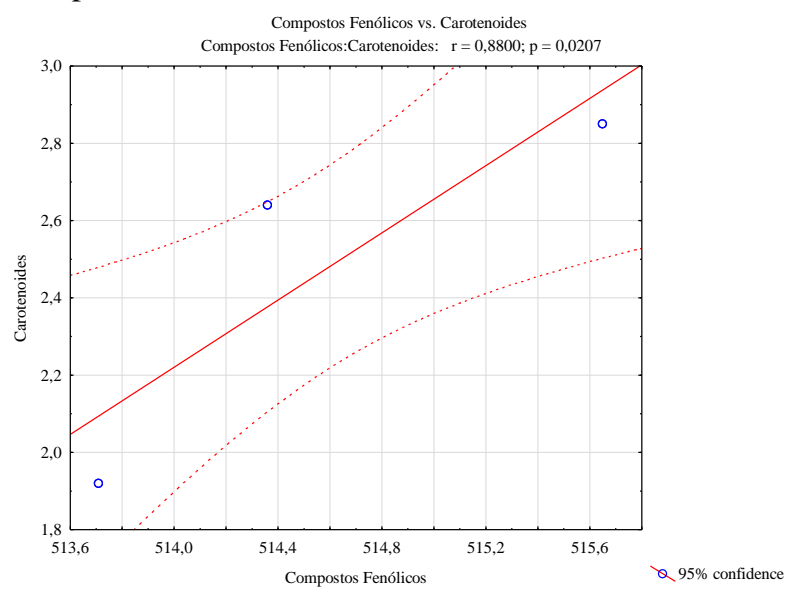

Já a correlação entre compostos fenólicos e antocianinas foi considerada moderada $(R=0,75)$ como pode ser observado na Figura 3. As antocianinas são pigmentos dissolvidos no tecido vegetal de flores e frutos, encontradas em abundancia em frutos como, mirtilo, cereja, morango e amora. O conteúdo de antocianinas presente nos frutos é proporcional a intensidade de coloração, as quais conferem cor rosa, vermelha, azul ou roxa (RIBEIRO, 2011).

Entre o conteúdo de fenólicos totais e flavonoides amarelos foi observada uma correlação moderada $(R=0,62)$, sugerindo que os flavonoides não são os principais componentes que conferem o potencial de fenólicos desse fruto, indicando presença de outros compostos.

Figura 3: Gráficos de Correlação entre o conteúdo de Compostos Fenólicos e Antocianinas.

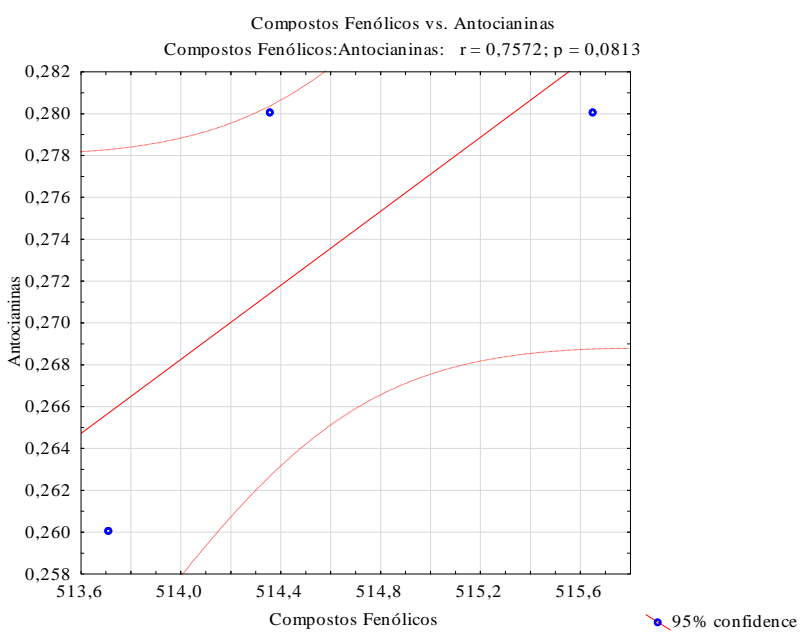

Entretanto, os resultados obtidos para a compostos fenólicos e o método de DPPH mostraram uma correlação fraca $(\mathrm{R}=0,18)$. Apesar deste método também estar baseado em mecanismos de ação semelhantes, ou seja, na transferência de elétrons.

Para os resultados entre conteúdo fenólico e o método de FRAP observou-se uma correlação considerada moderada $(\mathrm{R}=0,59)$ demonstrando um estreito vínculo entre os compostos fenólicos e o método de FRAP, indicando que a capacidade antioxidante da guapeva tem associação com os seus constituintes fenólicos.

Quanto ao teor de vitamina C existe uma forte correlação $(\mathrm{R}=0,83)$ com os carotenoides totais, demonstrada graficamente na Figura 4. Dados que corroboram com os resultados encontrados por Batista (2010) em estudo com os frutos acerola e goiaba.

Figura 4: Gráficos de Correlação entre o conteúdo de Vitamina C e Carotenoides. 




Em relação a vitamina $C$, pode-se observar uma moderada correlação com antocianinas e flavonoides $(\mathrm{R}=0,69$ e 0,55). Já a correlação de vitamina $C$ é fraca para o potencial antioxidante DPPH ( $\mathrm{R}=0,27)$, dados que corroboram com o estudo feito por Mahatanatawee et al. (2005) para diversos frutos tropicais com um coeficiente de correlação $(0,20)$.

Pode-se verificar que os carotenoides totais têm forte relação com antocianinas $(R=0,97)$ representado graficamente na Figura 5. Sabe-se que os carotenoides e as antocianinas se enquadram nos grupos de corantes naturas, que além de colorir apresentam atividade biológica, de forma a promover benefícios a saúde (MESQUITA et al, 2017). Este fato tem favorecido o uso de ambos na indústria alimentícia e também farmacêutica.

Figura 5: Gráficos de Correlação entre o conteúdo de Carotenoides e

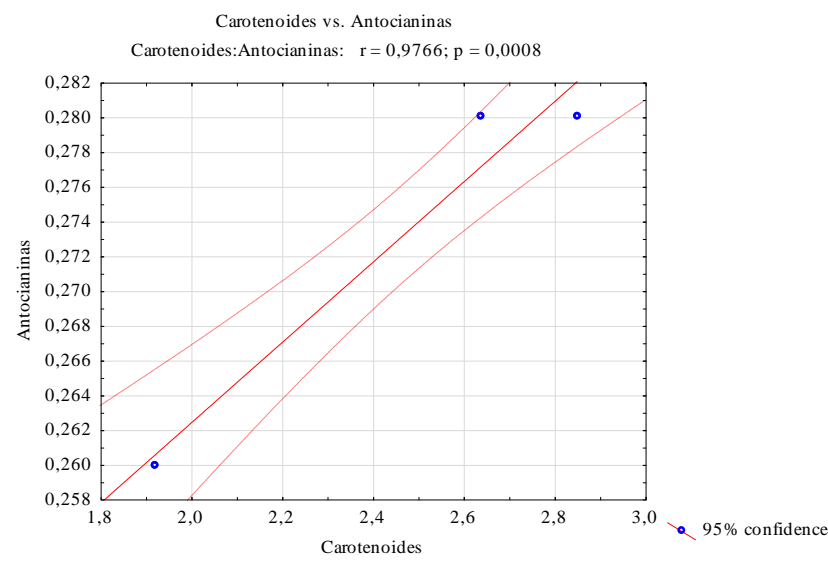

A antocianinas desenvolvem um importante papel nas plantas, dentre elas a capacidade antioxidante, ou seja, mecanismos de defesa em função biológica (LOPES et al., 2007).

No que se refere a carotenoides a correlação entre flavonoides demonstrou resultados que o classifica como forte correlação $(R=0,91)$, como pode ser observado na Figura 6 .

Figura 6: Gráficos de Correlação entre o conteúdo de Carotenóides e Flavonoides

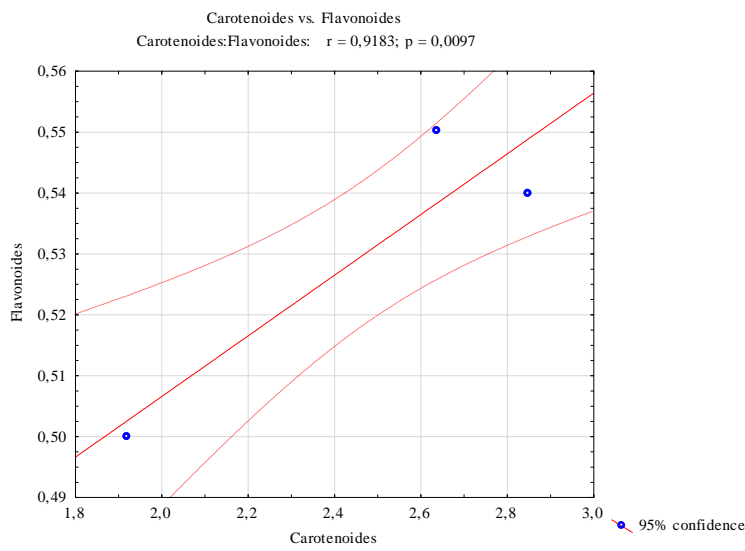

Assim como as antocianinas os flavonoides enquadram-se nos grupos de pigmento, sendo considerado juntamente com os carotenoides os principais antioxidantes distribuídos nas frutas e vegetais. Os flavonoides são apresentados sob muitas variações como flavonóis, flavonas, flavanonas, catequinas, antocianinas, isoflavonas e chalconas (SILVA et al., 2010). Isso deve explicar a forte correlação $(R=0,98)$ verificada entre as antocianinas e os flavonoides da guapeva (Figura7).

Figura 7: Gráficos de Correlação entre o conteúdo de Antocianinas e Flavonoides. 




Nos teores carotenoides totais observou-se uma correlação considerada fraca para as variáveis DPPH e FRAP que se justifica pela metodologia utilizada, segundo Oliveira et al. (2011) isso ocorre devido a polaridade constituída pelos sistemas, sendo mais indicadas para compostos hidrofílicos. Entretanto vale ressaltar que o potencial antioxidante de frutos não é resultante de um único componente fitoquímicos, mas do efeito sinergético de todos os compostos.

\section{CONCLUSÃO}

O fruto analisado contém compostos fenólicos, vitamina $\mathrm{C}$ e carotenóides com forte correlação, além de uma correlação moderada com FRAP. Deste modo o fruto é considerado como um alimento que possui um alto potencial antioxidante natural podendo ser incorporado na dieta humana, contribuindo para o consumo desses antioxidantes essenciais para a prevenção de doenças crônico degenerativas, tais como câncer.

\section{AGRADECIMENTOS}

A toda equipe do Laboratório de Cinética e Modelagem de Processos, e a Capes pelo apoio para o desenvolvimento dessa pesquisa.
Todos os autores declararam não haver qualquer potencial conflito de interesses referente a este artigo.

\section{REFERÊNCIAS}

ABRAHÃO, S. A. et al. Compostos bioativos e atividade antioxidante do café (Coffea arabica L.). Ciênc. agrotec., Lavras, v. 34, n. 2, p. 414-420, mar./abr., 2010

ALVES, L. F. Produção de Fitoterápicos no Brasil: História, Problemas e Perspectivas. Revista Virtual de Química. v. 5, n. 3, p. 450-513, 2013.

AMOROZO, M. C. M. e GÉLY, A.L. Uso de plantas medicinais por caboclos do Baixo Amazonas. Bol. Mus. Para. Emílio Goeldi., Ser. Bot. v.4, n.1, p.47131, 1988.

AMOROZO, M.C.M. Uso e diversidade de plantas medicinais em Santo Antônio do Leverger, MT, Brasil. Acta Bot. Bras. v.16, n.2, p.189-203, 2002.

BARBOSA, K. B. F. et al. Estresse oxidativo: conceito, implicações e fatores modulatórios. Revista de nutrição, 2010.

BRAND-WILLIAMS, W.; CUVELIER, M.-E.; BERSET, C. L. W. T. Use of a free radical method to evaluate antioxidant activity. LWT-Food science and Technology, v. 28, n. 1, p. 25-30, 1995.

BURGARD, D. R. Chemometrics: chemical and sensory data. CRC Press, 2018.

CANUTO, G. A. B., XAVIER, A. A. O., NEVES, L. C., \& BENASSI, M. D. T. (2010). Caracterização físico-química de polpas de frutos da Amazônia e sua correlação com a atividade anti-radical livre. Revista Brasileira de Fruticultura.

CAVALARI, T. G. F.; SANCHES, R. A. Os efeitos da vitamina C. Revista Saúde em Foco-Ano: 2018.

CETIN-KARACA, H.; NEWMAN, M. Antimicrobial efficacy of plant phenolic compound against Salmonella and Escherichia coli. Food Bioscience, v. 11, p. 8-16, 2015.

CONTRERAS-CALDERÓN, J. et al. Antioxidant capacity, phenolic content and vitamin $\mathrm{C}$ in pulp, peel and seed from 24 exotic fruits from Colombia. Food research international, v. 44, n. 7, p. 2047-2053, 2011.

CORDEIRO, J.M.P.; FÉLIX, L.P. Conhecimento botânico medicinal sobre espécies vegetais nativos da 
caatinga e plantas espontâneas no agreste da Paraíba, Brasil. Rev. Bras. Pl. Med. v.16, n.3, p.685-692, 2014.

FIGUEIREDO FILHO, D. B.; SILVA JÚNIOR, J. A. da. Desvendando os Mistérios do Coeficiente de Correlação de Pearson (r). Revista Política Hoje, Vol. 18, n. 1, 2009.

FRANÇA, I.S.X.; SOUZA, J.A.; BAPTISTA, R.S.; BRITTO, V.R.S. Medicina popular: benefícios e malefícios das plantas medicinais. Rev. Bras. Enferm. v.61, n.2, p.201-8, 2008.

FRANCIS, F. J.; MARKAKIS, P. C. Food colorants: anthocyanins. Critical Reviews in Food Science \& Nutrition, v. 28, n. 4, p. 273-314, 1989.

HIGBY, W. K. A simplified method for determination of some aspects of the carotenoid distribution in natural and carotene-fortified orange juice. Journal of Food Science, v. 27, n. 1, p. 42-49, 1962.

INSTITUTO ADOLFO LUTZ - IAL. Métodos físicoquímicos para análise de alimentos. 4. ed. São Paulo: IAL, 2008. $1020 \mathrm{p}$.

JARA-PALACIOS, M. J. et al. Assessment of white grape pomace from winemaking as source of bioactive compounds, and its antiproliferative activity. Food chemistry, v. 183, p. 78-82, 2015.

LAJILI, S. et al. Anti-inflammatory, analgesic activities and gastro-protective effects of the phenolic contents of the red alga, Laurencia obtusa. European Journal of Integrative Medicine, v. 8, n. 3, p. 298306, 2016.

LEE, S. Y. H .et al. The reaction of flavanols with nitrous acid protects against $\mathrm{N}$-nitrosamine formation and leads to the formation of nitroso derivatives which inhibit cancer cell growth. Free Radical Biology and Medicine, v. 40, n. 2, p. 323-334, 2006.

LOPES, T. et al. Antocianinas: uma breve revisão das características estruturais e da estabilidade. Current Agricultural Science and Technology, v. 13, n. 3, 2007.

MAHATANATAWEE, K.; GOODNER, K.; BALDWIN, E.; MANTHEY, J.; LUZIO, G. Total antioxidant activity of Florida's tropical fruit. Fist report for Trust Fd Project with Tropical Fruit Growers of South Florida. Winter Haven: USDA-ARS Citrus and Subtropical Products Laboratory, 2005.

MESQUITA, S. S.; TEIXEIRA, C. M. L. L.; SERVULO, E. F. C. Carotenoides: propriedades, aplicações e mercado. Revista virtual de Química, v. 9, n. 02, p. 672-688, 2017.

MOREIRA-ARAÚJO, R. S. dos R. et al. Bioactive compounds and antioxidant activity three fruit species from the Brazilian Cerrado. Revista Brasileira de Fruticultura, v. 41, n. 3, 2019.

MOTA, C. S. et al. Production of Pouteria gardneriana (A. DC.) Radlk. seedlings on different substrates. African Journal of Agricultural Research, v. 10, n. 30, p. 2961-2967, 2015.

OLIVEIRA, D. S. et al. Vitamina C, carotenoides, fenólicos totais e atividade antioxidante de goiaba, manga e mamão procedentes da Ceasa do Estado de Minas Gerais. Acta Scientiarum. Health Sciences, v. 33, n. 1, p. 89-98, 2011.

ROCHA, W. Si. et al. Compostos fenólicos totais e taninos condensados em frutas nativas do cerrado. Revista Brasileira de Fruticultura, v. 33, n. 4, p. 1215-1221, 2011.

RUFINO, M. D. S. M. et al. Metodologia científica: determinação da atividade antioxidante total em frutas pelo método de redução do ferro (FRAP). Embrapa Agroindústria Tropical-Comunicado Técnico (INFOTECA-E), 2006. 
RUFINO, M. do S. M. et al. Metodologia Científica: Determinação da Atividade Antioxidante Total em Frutas pela Captura do Radical Livre DPPH. Comunicado Técnico 127. Embrapa, Fortaleza, 2007.

SILVA, M. L. C. et al. Compostos fenólicos, carotenóides e atividade antioxidante em produtos vegetais. Semina: Ciências Agrárias, v. 31, n. 3, p. 669-681, 2010.

SINGLETON, V. L.; ROSSI, J. A. Colorimetry of total phenolics with phosphomolybdicphosphotungstic acid reagents. American journal of Enology and Viticulture, v. 16, n. 3, p. 144-158, 1965.

SOUZA, A. V. de et al. Correlações entre compostos fenólicos e atividade antioxidante em casca e polpa de variedades de uva de mesa. Brazilian Journal of Food Technology, 2018.

TURNER, F.J. WERHOOGEN, J. Igneous and Metamorphic Petrology. 20a ed., McGraw-Hill, 694 p., 1960.

VÍTOLO, H. F.; SOUZA, G. M.; SILVEIRA, J. A. G. Cross-scale multivariate analysis of physiological responses to high temperature in two tropical crops with $\mathrm{C} 3$ and $\mathrm{C} 4$ metabolism. Environmental and Experimental Botany, v. 80, p. 54-62, 2012. 